\title{
AN OPTIMISED SUPPORT VECTOR MACHINE MODEL FOR ELASTIC MODULUS PREDICTION OF CONCRETE SUBJECT TO ALKALI SILICA REACTION
}

\author{
T.N. Nguyen, Y. Yu*, J. Li* and V. Sirivivatnanon \\ School of Civil and Environmental Engineering, University of Technology Sydney, NSW 2007, Australia \\ Emails: thucnhu.nguyen@student.uts.edu.au,yang.yu@uts.edu.au,jianchun.li@uts.edu.au, \\ vute.sirivivatnanon@uts.edu.au \\ *Corresponding authors
}

\begin{abstract}
Alkali-silica reaction (ASR) can induce the damage and loss in serviceability of concrete structures. Many studies have been conducted to investigate the influence of ASR on the degradation of mechanical properties of the concrete. Their results show that compared with other mechanical properties, the modulus of elasticity is the most affected by ASR, where the reduction is up to roughly $70 \%$ compared to its properties without expansion. In this study, to effectively assess the reduction of the modulus of elasticity caused by ASR, a novel predictive model is proposed based on support vector machine (SVM), in which the mix proportion of concrete, exposure environment and corresponding expansion are employed as the inputs and the output is the modulus of elasticity degradation. To improve the generalization capacity of the proposed predictive model, three different optimization algorithms are adopted to select optimal model parameters. Finally, the experimental data from the existing literatures are used to test the performance of the proposed method with satisfactory results.
\end{abstract}

Keywords: Alkali-silica reaction; elastic modulus; support vector machine; optimization algorithms

\section{INTRODUCTION}

Alkali-silica reaction (ASR) is a chemical reaction between alkali hydroxides in the concrete pore solution and reactive minerals within the aggregates. The consequences are the initiation of cracks in the reactive aggregate particles, propagation of the cracks into the paste matrix, exudation of the gel from the cracks, volumetric expansion of the concrete, stress development due to expansive pressure, and changes of mechanical properties.

Due to the importance of the mechanical properties during condition assessment and load capacity evaluation of the ASR-affected concrete structures, many studies have been conducted to investigate changes of strength and stiffness in relation to ASR-induced expansion (Sanchez et al. 2017; Smaoui et al. 2005). Most of these studies agreed that the elastic modulus presents significant reduction compared to the splitting tensile strength and compressive strength. The elastic modulus has been thus commonly considered as an indicator of ASR-affected concrete deterioration (Esposito et al. 2016; Sanchez et al. 2017). Furthermore, different analytical models represent relationship between elastic modulus and expansion level have been proposed to estimate the stiffness for damage assessment and load capacity evaluation (Esposito et al. 2016; ISE 1992; Kawabata et al. 2017).

Yet, both the ASR-induced expansion and changes mechanical properties of ASR-affected concrete are influenced by many factors from mix proportions, reactive aggregates, and 
environment conditions (Esposito et al. 2016; Giaccio et al. 2008; Sanchez et al. 2017; Smaoui et al. 2005). According to Helene, Carvalho \& Pacheco (2017), there are five main factors that affect to the deterioration intensity of ASR-affected concrete, including (1) cement alkali content and cement content, (2) alkali content from other sources such as from aggregates and external addition of alkalies, (3) number, size and reactivity of the aggregate, (4) moisture condition in concrete and (5) temperature condition. Therefore, the proposed analytical curves can only indicate the relationship in a particular set of the factors, or present a conservative curve as lower bounds. Meanwhile, the change of mechanical properties at an expansion level shows a significant variation which due to the effect of different factors. This issue brings challenges to estimate the elastic modulus change due to ASR based on a comprehensive information of testings.

This paper aim to improve the estimation of elastic modulus of ASR-affected concrete based on not only expansion but also other affected factors by means of soft-computing techniques. The support vector machine (SVM) with three different optimization algorithms are adopted to assess the reduction of the modulus of elasticity with large number of input parameters.

\section{DATA SET}

A number of factors affects the relationship between the elastic modulus and ASR induced-expansion levels. In addition to the expansion, other factors that affect to the mechanism of ASR and concrete properties were collected, including the mix proportion, proportion of reactive sand and coarse aggregate, exposure condition (temperature and moisture), equivalent proportion of $\mathrm{Na} 2 \mathrm{O}$ for ASR acceleration, initial compressive strength at 28-day. To develop a high accuracy model, a large number of data samples are necessary. In this study, the data were obtained from a comprehensive literature review. The dataset was collected from 12 studies with more than 38 concrete mixes, including 196 testing groups for modulus of elasticity at different levels of ASR-induced expansion (Ahmed et al. 2003; Esposito et al. 2016; Gautam et al. 2017; Giaccio et al. 2008; Kubo \& Nakata 2012; Larive 1997; Mohammed, Hamada \& Yamaji 2003; Multon 2003; Pleau et al. 1989; Sanchez 2014; Sargolzahi et al. 2010; Smaoui et al. 2005). Table 1 shows the detail of each parameter and their variation.

Table 1 Model parameters and variation range

\begin{tabular}{|c|c|c|c|c|}
\hline Type & Parameter description & Symbol & Unit & $\begin{array}{l}\text { Variation range } \\
{[\min , \max ]}\end{array}$ \\
\hline Input & $\begin{array}{l}\text { 1. Cement content } \\
\text { 2. Water/cement ratio } \\
\text { 3. Fine reactive aggregate / cement ratio } \\
\text { 4. Coarse reactive aggregate / cement ratio } \\
\text { 5. Total reactive aggregate / cement ratio } \\
\text { 6. Non-reactive aggregate / cement ratio } \\
\text { 7. Exposure temperature } \\
\text { 8. Relative humidity } \\
\text { 9. Equivalent alkali content } \\
\text { 10. Initial compressive strength at "non-expansive" } \\
\text { 11. Maximum measured expansion } \\
\text { 12. Measured expansion }\end{array}$ & $\begin{array}{l}\text { C } \\
\text { WC } \\
\text { FRA } \\
\text { CRA } \\
\text { RA/C } \\
\mathrm{NRC} \\
\mathrm{T} \\
\mathrm{M} \\
\mathrm{Na}_{2} \mathrm{O} \\
\mathrm{Fc} \\
\mathrm{EM} \\
\text { Exp }\end{array}$ & $\begin{array}{l}\mathrm{Kg} \\
- \\
- \\
- \\
- \\
{ }^{\circ} \mathrm{C} \\
\% \\
\% \\
\mathrm{MPa} \\
\% \\
\%\end{array}$ & $\begin{array}{l}{[300,424]} \\
{[0.370,0.611]} \\
{[0,2.85]} \\
{[0,3.42]} \\
{[0,6.13]} \\
{[0,4.02]} \\
{[38,50]} \\
{[95,100]} \\
{[1.17,2.87]} \\
{[18.2,58.5]} \\
{[0.072,0.916]} \\
{[0.001,0.916]}\end{array}$ \\
\hline Output & Normalised elastic modulus & Ec & - & {$[0.163,1.130]$} \\
\hline
\end{tabular}




\section{PROPOSED LEARNING MODEL FOR ELASTICI MODULUS PREDICTION}

\subsection{Introduction of Support Vector Machine}

Support vector machine (SVM) is regarded as a powerful method to deal with the classification and regression problems, which is based on the structural risk minimization principle (Yu et al. 2015; 2016; 2018). So far, SVM has been successfully applied to the field of nonlinear system modelling. The main idea of SVM is illustrated as: Suppose there is a given training set $\mathrm{S}=\left[\left(\mathrm{x}_{\mathrm{i}}, \mathrm{y}_{\mathrm{i}}\right), \mathrm{i}=1,2, \ldots, 1\right]$, where $\mathrm{x}_{\mathrm{i}}$ and $\mathrm{y}_{\mathrm{i}}$ denote the input vector and the output (target) value, respectively; 1 is the total number of the data pattern. The objective of SVM is to design a regression model to perfectly portray the nonlinear relationship between input and output variables, and forecast the output result according to the new input samples. The expression of SVM function can be described by:

$$
\begin{aligned}
& f(x)=w \phi(x)+b \\
& \phi: R^{n} \rightarrow F, w \in F
\end{aligned}
$$

where $\phi(\mathrm{x})$ is a nonlinear function in the high-dimensional characteristic space; $\mathrm{w}$ and $\mathrm{b}$ denote coefficients. To obtain $\mathrm{w}$ and $\mathrm{b}$, two positive slack variables $\xi_{\mathrm{i}}$ and $\xi_{i}^{*}$ are introduced so that the problem is transformed to the following optimization problem:

$$
\begin{array}{ll}
\min & \frac{1}{2}\|w\|^{2}+C \sum_{i=1}^{l}\left(\xi_{i}+\xi_{i}^{*}\right) \\
\text { s.t. } \quad & y_{i}-w \phi\left(x_{i}\right)-b \leq \varepsilon+\xi_{i} \\
& w \phi\left(x_{i}\right)+b-y_{i} \leq \varepsilon+\xi_{i}^{*}, \xi^{(*)} \geq 0
\end{array}
$$

Eventually, the optimal nonlinear regression function (hyper-plane) can be obtained by utilizing above minimization function:

$$
f(x)=\sum_{i=1}^{n}\left(\alpha_{i}-\alpha_{i}^{*}\right) k\left(x_{i}, x\right)+b
$$

where $\mathrm{k}\left(\mathrm{x}_{\mathrm{i}}, \mathrm{x}\right)$ denotes the kernel function, which plays an important role in building the optimal hyper-plane. The conventional kernel functions include linear function, polynomial function, radial basis function and sigmoid function. Different kernel functions have different parameters, which correlate the configuration of the feature space as well as the solution complexity.

\subsection{SVM Parameter Analysis}

To develop a high accuracy predictive model for elastic modulus prediction of ASRaffected concrete, the parameters of the SVM should be set properly. Improper values of parameters may result in under-fitting or over-fitting. Different parameter combination could lead to noticeably distinct performance of trained models. Hence, searching for optimal values of parameters is a significant step in devising the SVM model. Here, the SVM parameters are: (i) regularization parameter $C$, which is used to adjust the trade-off cost between the complicity of the model and the training error; (ii) and $\varepsilon$-insensitive loss function parameter $\gamma$, which is used to tune the size of the $\varepsilon$-insensitive region. The value of $\gamma$ is able to influence the support vector's number to build the regression function.

\subsection{Proposed optimised SVM model}

In this paper, an SVM-based predictive model is built up to forecast the elastic modulus of concrete due to ASR. The configuration of the proposed model is given in Figure 1. In which the model inputs are cement content, ratio of water to cement, fine reactive aggregate content, coarse reactive aggregate content, ratio of reactive aggregate to cement, ratio of nonreactive aggregate to cement, temperature, humidity, $\mathrm{Na}_{2} \mathrm{O}$ content, compressive strength at 28 days, 
elastic modulus at 28 days and expansion caused by ASR and the output of the model is the reduction of the elastic modulus. To obtain the optimal performance of the developed model, three different optimization algorithms, particle swarm optimization (PSO), genetic algorithm (GA) and grid search (GS), are employed to select optimal model parameters during the training procedure. Therefore, a reasonable fitness function is required to implement this optimization problem. Here, the mean squared error (MSE) between the real values of elastic modulus reduction and the SVM predictions are adopted as the fitness function in this work, and its mathematical expression is given as follows.

$$
\text { Fit }=\frac{1}{N} \sum_{i=1}^{N}\left[E M_{r}(i)-E M_{p}(i)\right]^{2}
$$

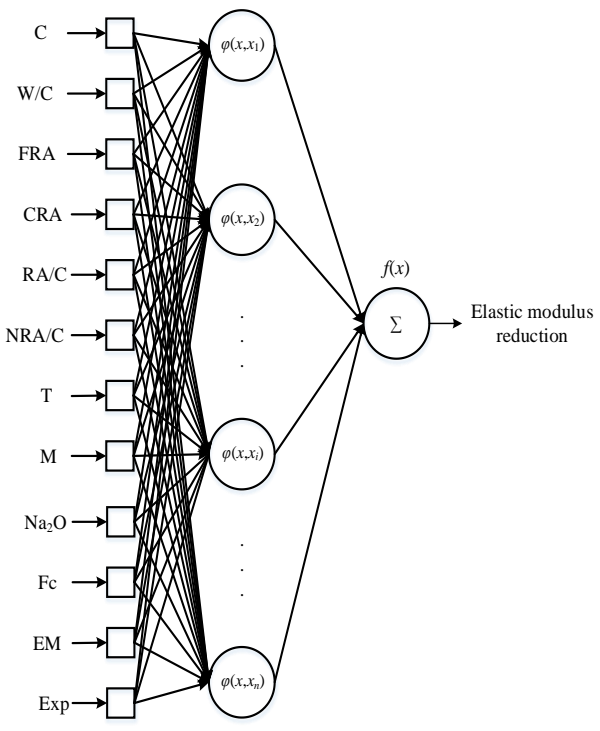

(a)

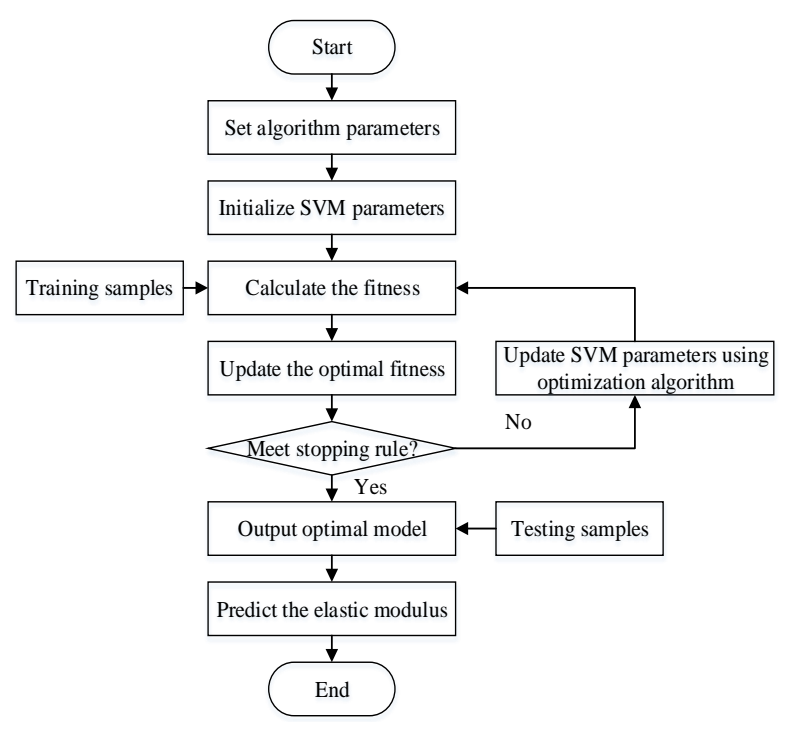

(b)

Figure 1. (a) Configuration of the proposed SVM model, and (b) Flowchart of training and validation of the developed SVM model for elastic modulus prediction

where Fit denotes the fitness function value, $E M_{r}$ denotes the real elastic modulus reduction, $E M_{p}$ denotes the predicted elastic modulus reduction, and $N$ denotes the number of training samples. The detailed procedure of optimized SVM model for elastic modulus prediction of ASR affected concrete is provided in Figure 1 (b). First, set the optimization algorithm parameters and initialize the SVM model parameters. Here, the model parameters include regularization parameter and $\varepsilon$-insensitive loss function parameter. Then, based on the initial values and training samples, calculate the fitness value of the optimization problem. Judge the stopping criterion, if the criterion is satisfied, terminate the model training and output the optimal model for validation based on testing data. If the criterion is not satisfied, use the optimization algorithms to update the model parameters and then calculate the fitness value. Compare the current fitness with previous ones, if the current value is better than previous one, update the model with optimal parameters; otherwise, keep the model unchanged.

\section{RESULTS AND ANALYSIS}

The SVM model and optimization algorithms are implemented using Matlab V.2012b. The optimization algorithms parameters are set according to ( $\mathrm{Yu}$ et al 2015). The ranges of the SVM parameters are set as $[0,100]$. All the experimental data are randomly divided into two groups: training group and testing group. The data in the training group are used to set up the predictive model while the data in the testing group is employed to validate the performance of the trained model. In this study, $70 \%$ of the data samples are used for training and the rest 
data are used for testing. To avoid the error due to the different dimensions of input variables, all the training and testing data are normalized before the model training. For an $m \times n$ matrix, all the elements in the matrix will be scaled into $[0,1]$ using the following equation:

$$
x_{i}^{\text {norm }}=\frac{x_{i}-x_{\min }}{x_{\max }-x_{\min }}
$$

where $x_{i}$ is the $i$ th raw data, and $x_{\max }$ and $x_{\min }$ denote the maximal and minimal values of the data set.

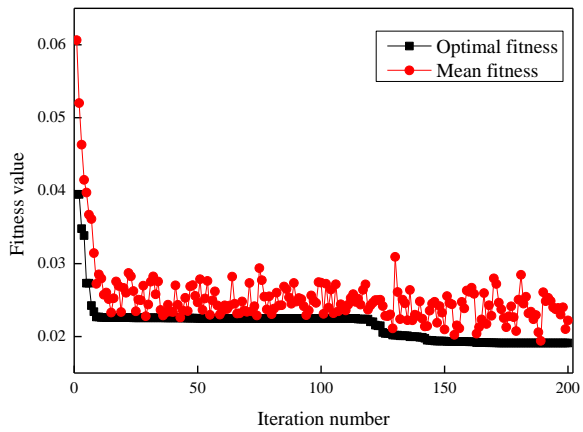

(a) Fitness change

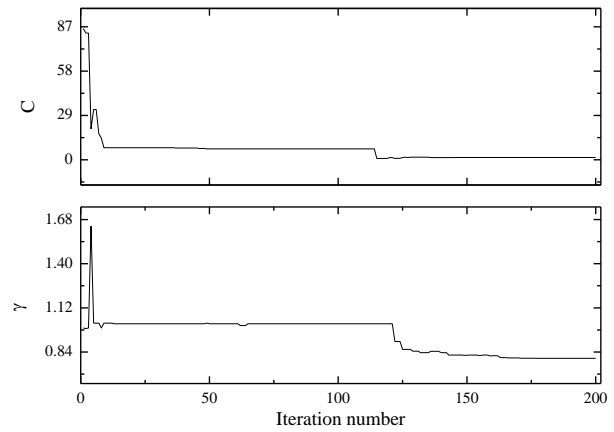

(b) parameter change

Figure 2 SVM model optimization using PSO

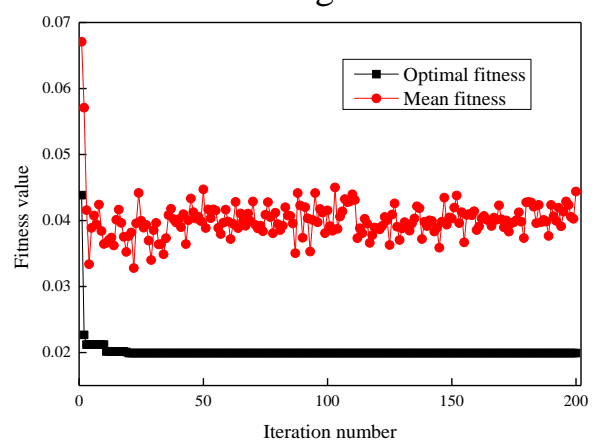

(a) Fitness change
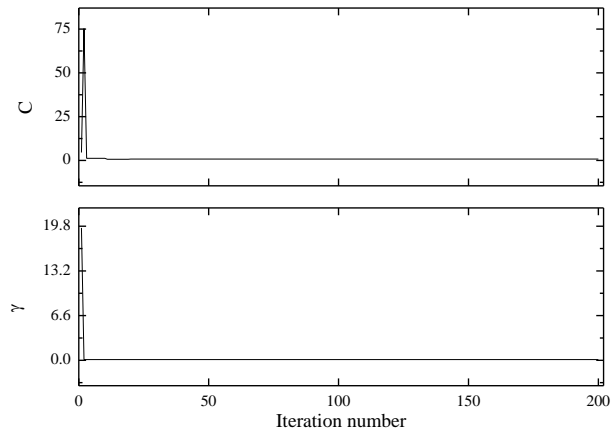

(b) parameter change

Figure 3 SVM model optimization using GA

Based on the training data, the SVM is developed via model training with parameter optimization. Figures show the effectiveness of three different optimization algorithms for SVM parameters. Figure 2 shows the optimization results using PSO algorithm. It can be seen that optimal SVM parameters $\mathrm{C}$ and $\gamma$ are 1.1498 and 0.8001 , respectively. And the algorithm converges at around 140 iterations. Figures 3 shows the SVM optimization using GA. It is noticeable that compared with PSO, the GA arrives at the optimal values of optimization problem much faster, which indicates that the algorithm may fall into the local optimum. The corresponding optimal parameter values are 0.7540 and 0.1000 , respectively. Figure 4 describes the 2-D and 3-D contour of SVM parameter optimization using GS method. It can be observed from the figure that both maximal contour lines arrive at the same value of $99 \%$, which means that both lines have the perfect accuracy. And, the corresponding parameter values of the SVM model are 64 and 0.1436 , respectively.
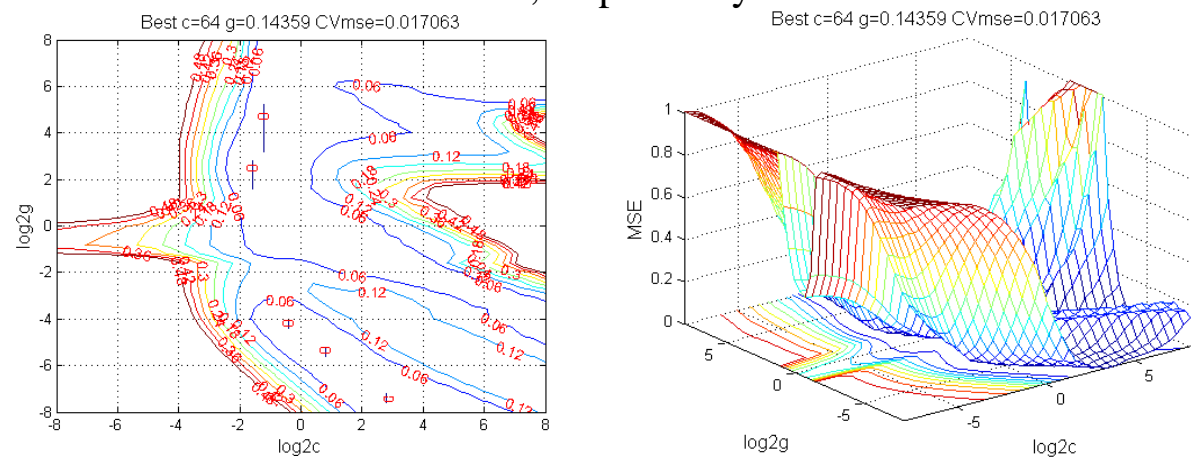


\section{$\begin{array}{ll}\text { (a) 2-D contour } & \text { (b) 3-D contour }\end{array}$}

Figure 4 SVM model optimization using GS

Figures 5-7 provides the comparison between real values of elastic modulus and the SVM predicted values for both training and validation samples. It is clearly seen that the SVM models with different parameter optimizations are capable of accurately forecast the elastic modulus reduction of concrete caused by ASR effect with the various concrete information. Even though several obvious errors happen at some specific points, the optimized SVM model satisfactorily track the elastic modulus change, which means that it is applicable for predicting how different input variables can affect the elastic modulus variation.

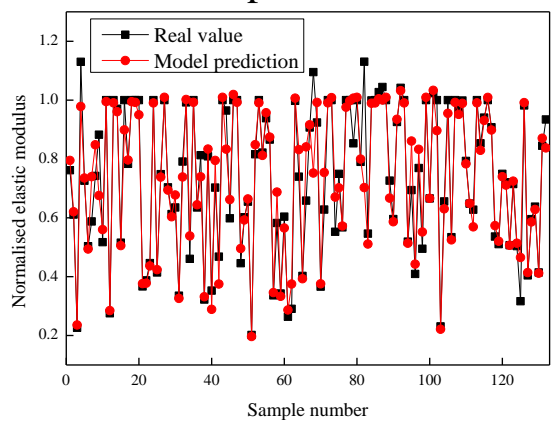

(a) Training

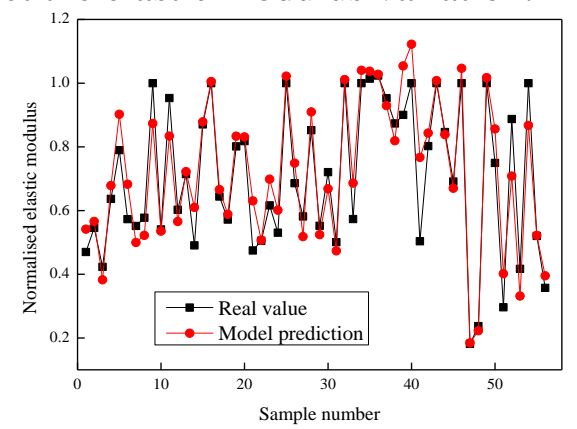

(b) Validation

Figure 5 Comparison between real and predicted elastic modulus using PSO-SVM

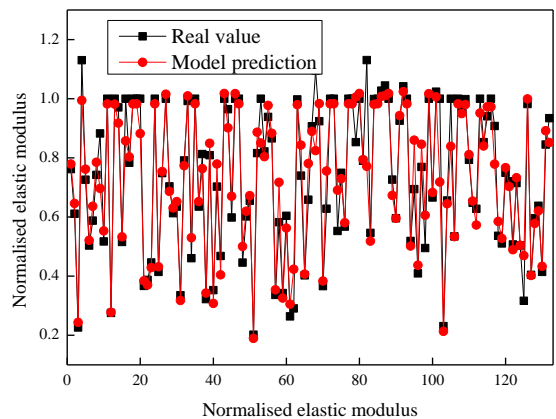

(a) Training

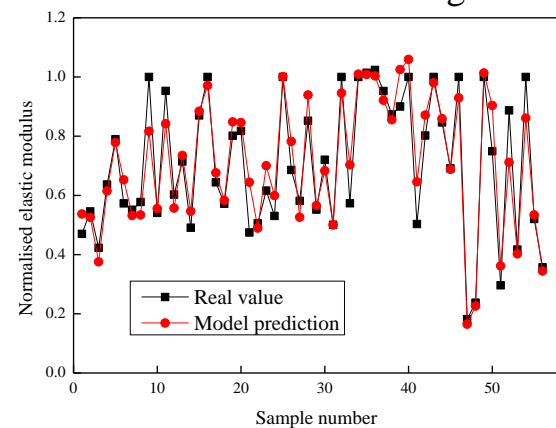

(b) Validation

Figure 6 Comparison between real and predicted elastic modulus using GA-SVM

To further validate the performance of three SVM models for the prediction of the elastic modulus reduction, the correlation analysis is conducted for both training and validation samples, respectively. The analysis results are shown in Figures 8-10. It is clearly noted that most data points evenly scatter near the fitted line $y=x$ for three models. The closer the distances between the points and the regression line are, the more accurate the model is. According to the correlation analysis results, for the training data three models are able to provide similar identification accuracy with the correlation coefficient around 0.95. However, for the testing date, the PSO-SVM outperforms the GA-SVM and GS-SVM models in terms of the correlation coefficient. For the PSO-SVM model, the correlation coefficient is 0.9499, while for the GA-SVM and GS-SVM models, the corresponding correlation coefficients are 0.9400 and 0.9284 , respectively. The main reason contributing to this phenomenon is that both GA and GS algorithms may fall into the local optimum, which results in the premature convergence and affects the identification accuracy. Overall, the results obtained from three models can be acceptable because all the correlation coefficients are above 0.9. Accordingly, the proposed SVM with parameter optimizations is more reliable and can be used to predict the elastic modulus reduction of ASR-affected concrete structures in practice. 


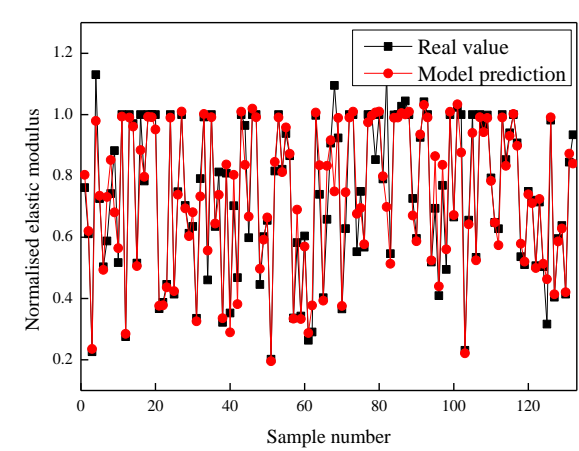

(a) Training

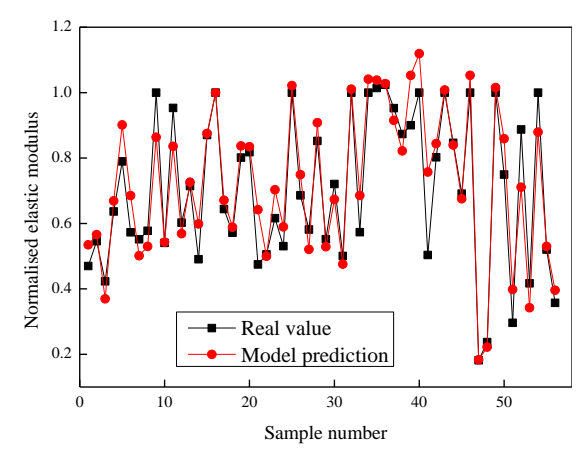

(b) Validation

Figure 7. Comparison between real and predicted elastic modulus using GS-SVM

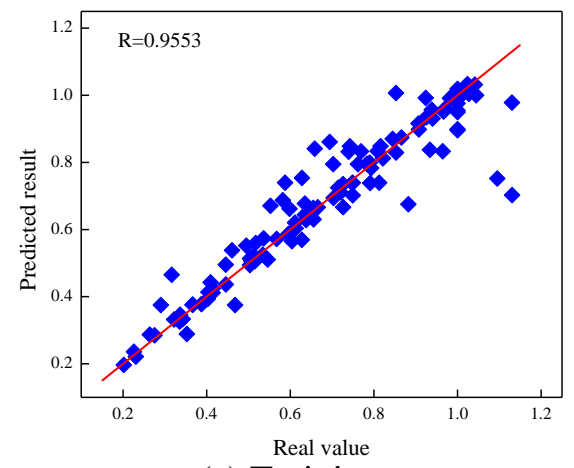

(a) Training

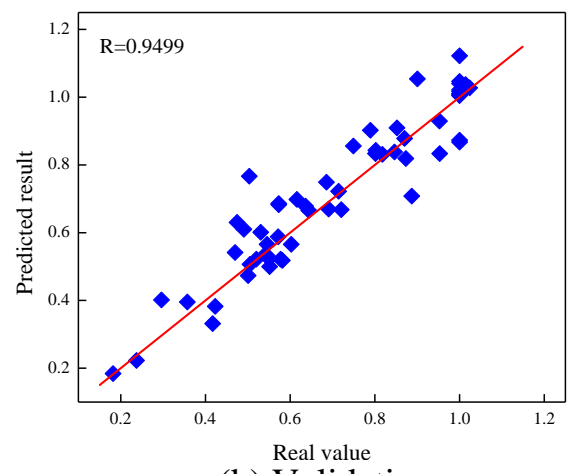

(b) Validation

Figure 8 Correlation analysis of prediction results using PSO-SVM.

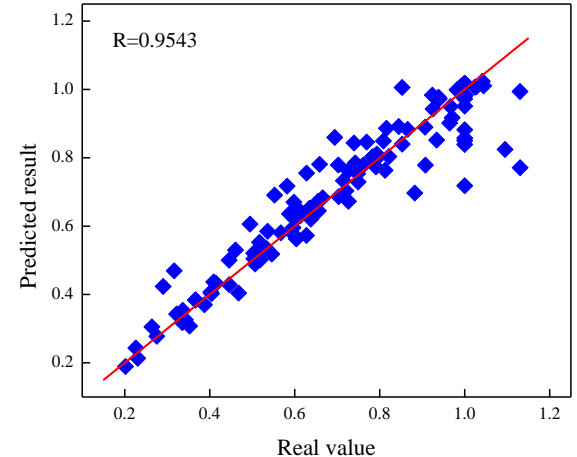

(a) Training

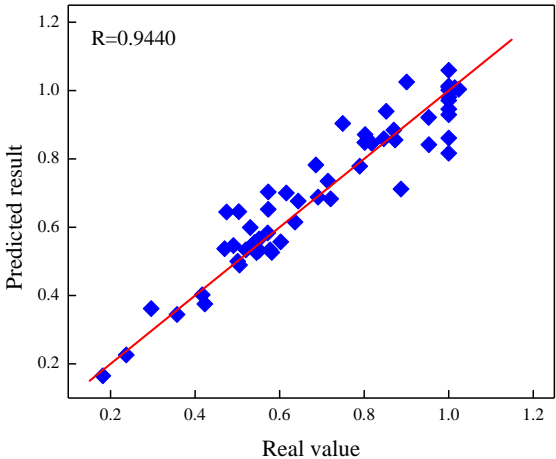

(b) Validation

Figure 9 Correlation analysis of prediction results using GA-SVM.

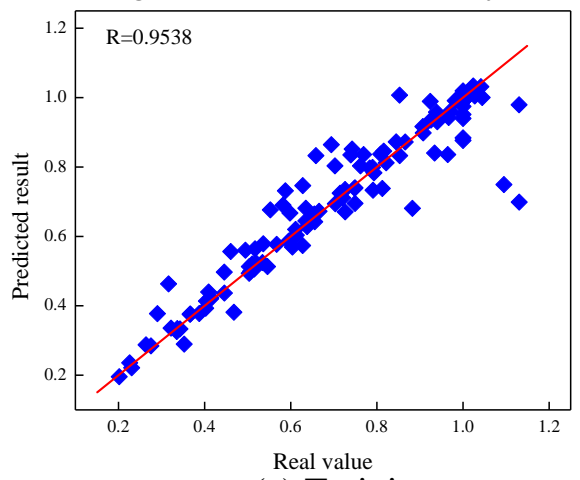

(a) Training

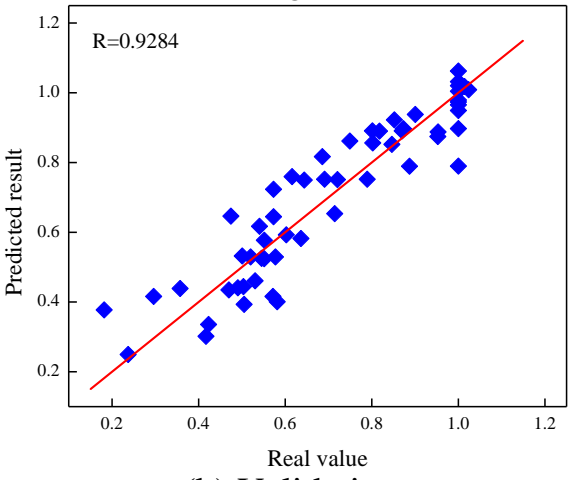

(b) Validation

Figure 10 Correlation analysis of prediction results using GS-SVM.

\section{CONCLUSION}

This paper numerically investigates the mechanical property of the concrete affected by ASR, which can cause the concrete expansion and subsequent degradation of the modulus of 
elasticity. A predictive model based on soft computing technique is developed to forecast this property. To improve the model recognition accuracy, three different optimisation algorithms, PSO, GA and GS, are employed to optimise the model parameters. Finally, the experimental data collected from published literatures are used to evaluate the performance of the optimised model. The results show that the model optimised by PSO is capable of providing the optimal result with the highest correlation coefficient of 0.95 for validation samples.

\section{ACKNOWLEDGEMENT}

This work was supported by the Australian Research Council Research Hub (IH150100006) for Nanoscience Based Construction Materials Manufacturing (NANOCOMM) and the industry partner Roads and Maritime Services (RMS).

\section{REFERENCES}

Ahmed, T., Burley, E., Rigden, S., Abu-Tair, A.I. (2003), The effect of alkali reactivity on the mechanical properties of concrete, Construction and Building Materials, 17(2), 123-144.

Esposito, R., Anaç, C., Hendriks, M.A., Çopuroğlu, O. (2016), Influence of the alkali-silica reaction on the mechanical degradation of concrete, Journal of Materials in Civil Engineering, 28(6), 04016007.

Gautam, B.P., Panesar, D.K., Sheikh, S.A., Vecchio, F.J. (2017), Effect of coarse aggregate grading on the ASR expansion and damage of concrete, Cement and Concrete Research, 95, 75-83.

Giaccio, G., Zerbino, R., Ponce, J., Batic, O.R. (2008), Mechanical behavior of concretes damaged by alkalisilica reaction, Cement and Concrete Research, 38(7), 993-1004.

Helene, P., Carvalho, M., Pacheco, J. (2017), 'Engineering field tests for alkali-aggregate reaction', Structural Concrete, vol. 18, no. 2, pp. 349-355.

ISE (1992), Structural effects of alkali-aggregate reaction: technical guidance on the appraisal of existing structures.

Kawabata, Y., Seignol, J.-F., Martin, R.-P., Toutlemonde, F. (2017), Macroscopic chemo-mechanical modeling of alkali-silica reaction of concrete under stresses, Construction and Building Materials, 137, 234-45.

Kubo, Y., Nakata, M. (2012), Effect of Reactive Aggregate on Mechanical Properties of Concrete Affected by Alkali-Silica Reaction, 14th International conference on alkali-aggregate reaction. Texas, USA.

Larive, C. (1997), Apports combinés de l'expérimentation et de la modélisation à la compréhension de l'alcaliréaction et de ses effets mécaniques, Ecole nationale des ponts et chaussees.

Mohammed, T.U., Hamada, H.,Yamaji, T. (2003), Relation between Strain on Surface and Strain over Embedded Steel Bars in ASR Affected Concrete Members, Journal of Advanced Concrete Technology, 1(1), 76-88.

Multon, S. 2003, 'Evaluation expérimentale et théorique des effets mécaniques de l'alcali-réaction sur des structures modèles', Marne-la-Vallée.

Pleau, R., Bérubé, M., Pigeon, M., Fournier, B. \& Raphaël, S. 1989, 'Mechanical behaviour of concrete affected by ASR', Proc., 8th Int. Conf. on Alkali-Aggregate Reaction, Soc. of Mat. Sci., Kyoto, Japan, pp. 721-6.

Sanchez, L. 2014, 'Contribution to the assessment of damage in aging concrete infrastructures affected by alkaliaggregate reaction', Citeseer.

Sanchez, L.F.M., Fournier, B., Jolin, M., Mitchell, D. \& Bastien, J. 2017, 'Overall assessment of AlkaliAggregate Reaction (AAR) in concretes presenting different strengths and incorporating a wide range of reactive aggregate types and natures', Cement and Concrete Research, vol. 93, pp. 17-31.

Sargolzahi, M., Kodjo, S.A., Rivard, P., Rhazi, J. (2010), Effectiveness of nondestructive testing for the evaluation of alkali-silica reaction in concrete, Construction and Building Materials, 24(8), 1398-403.

Smaoui, N., Bissonnette, B., Bérubé, M.-A., Fournier, B, Durand, B. (2005), Mechanical properties of ASRaffected concrete containing fine or coarse reactive aggregates, ASTM International, 3(3), 1-16.

Yu, Y., Li, Y., Li, J. (2015), Forecasting hysteresis behaviours of magnetorheological elastomer base isolator utilizing a hybrid model based on support vector regression and improved particle swarm optimization, Smart Materials and Structures, 24(3), 035025.

Yu, Y., Li, Y., Li, J., Gu, X. (2016), A novel optimised self-learning method for compressive strength prediction of high performance concrete, Neurocomputing, 211, 41-52.

Yu, Y., Li, W., Li, J., Nguyen, T. (2018), A novel optimised self-learning method for compressive strength prediction of high performance concrete, Construction and Building Materials, 184, 229-47. 